\title{
Modification of the refractive index response of long period gratings using thin film overlays
}

\author{
Imran M. Ishaq*, Antonio Quintela ***, Stephen W. James*, Geoffrey J. Ashwell**, \\ José M. Lopez-Higuera*** and Ralph P. Tatam* \\ *Optical Sensors Group and ${ }^{* *}$ Nanomaterials Group , Centre for Photonics and Optical \\ Engineering, School of Engineering, Cranfield University, Bedford MK43 0AL, UK \\ *** Grupo de Ingenieria Fotonica-Universidad de Cantabria, \\ E.T.S.I.I. y Telecommunicacion, Santander, Spain
}

\begin{abstract}
The response of a fibre optic long period grating (LPG) to the surrounding refractive index is shown to be modified by the deposition of a thin film of thickness of order $100 \mathrm{~nm}$ onto the cladding. The LPG becomes sensitive to surrounding refractive indices greater than that of silica, and exhibits an enhanced sensitivity to external refractive indices lower than that of silica.
\end{abstract}


Fibre optic long period grating (LPG) devices are being developed and investigated for a range of uses in telecommunications and sensing systems ${ }^{1,2}$. The sensitivity of the transmission spectrum of a LPG to the refractive index of the surrounding material has been extensively investigated and has been exploited for a range of applications, including, for example, as a refractive index sensor ${ }^{3}$, a chemical solution concentration sensor ${ }^{4,5,6}$, to form a liquid level sensor ${ }^{7}$, as a means of controlling the thermal response of the $\mathrm{LPG}^{8}$ and for forming a tunable spectral filter ${ }^{9}$. While LPGs formed in silica based optical fibres are sensitive over only a limited refractive index range, typically from 1.4 to 1.45 , and their response is not species specific, it has been shown that LPGs may be used for on-line monitoring of the concentration of aqueous solutions of materials especially in inaccessible or hazardous locations for industrial production quality control ${ }^{6}$. Concentrations of solutions of sodium chloride ${ }^{6}$, calcium chloride ${ }^{6}$ and ethylene glycol ${ }^{5,6}$ have been measured. In this paper the use of a thin film overlay, deposited around the fibre, concentric with the LPG, to modify the range of refractive indices to which the LPG is sensitive is explored, extending their chemical concentration measurement capabilities.

An LPG is a periodic modulation of the refractive index of the core of an optical fibre. The period of the modulation is typically in the range $10 \mu \mathrm{m}$ to $1000 \mu \mathrm{m}$. The correspondingly small grating wave-vector promotes coupling between co-propagating modes of the optical fibre. In the case of single mode fibre, the coupling takes place between the guided mode and co-propagating cladding modes. Efficient coupling is possible only at discreet wavelengths ${ }^{10}$. As the cladding modes suffer from high attenuation, the transmission spectrum of an optical fibre containing an LPG contains a number of attenuation bands, each corresponding to coupling to a different cladding mode, as is shown in figure 1. 
The attenuation bands are centred on phase matching wavelengths governed by the expression $^{11}$

$$
\lambda_{i}=\left[n_{e f f}(\lambda)-n_{c l}^{i}(\lambda)\right] \Lambda
$$

where $n_{\text {eff }}(\lambda)$ is the effective refractive index of the propagating core mode at wavelength $\lambda_{\mathrm{i}}$, $n_{c l}^{i}(\lambda)$ is the refractive index of the $i^{\text {th }}$ cladding mode and $\Lambda$ is the period of the LPG. The refractive index sensitivity of the transmission spectrum of an LPG arises from the dependence of the phase matching wavelengths upon the cladding mode effective index, which is dependent upon the refractive index of the surrounding material.

The form of the refractive index sensitivity of an LPG fabricated in B/Ge co-doped photosensitive optical fibre (Fibrecore PS 750) is shown in figure 2, where the shift of the central wavelength of the $5^{\text {th }}$ attenuation band in the spectrum of the LPG when the submerged in a series of external media of different refractive indices is plotted. It is apparent the LPG's sensitivity lies predominantly within the range 1.400 to 1.456 . The upper limit is imposed by the refractive index of the cladding material. For refractive indices above this limit there is no measurable wavelength response.

Previously we have shown that the transmission spectrum of an LPG is highly sensitive to the optical thickness of an overlay material deposited onto the optical fibre ${ }^{12}$. Here we aim to extend this study and investigate the effect of the presence of such an overlay material upon the response of the LPG to the surrounding refractive index. 
A theoretical analysis of an LPG's response to the presence of the thin film overlay has been performed, allowing the dependence of the central wavelengths of the attenuation bands upon the refractive index of the surround material, both with, and without, a thin film overlay, to be determined. The effective refractive indices of the cladding modes were calculated as a function of wavelength and overlay thickness by considering the cladding/ overlay system as a stack of thin films and employing the transfer matrix method ${ }^{13}$. The effective refractive index of the propagating core mode was calculated using the weakly guiding approximation of Gloge $\mathrm{e}^{14}$. Using the calculated dispersion of the core and cladding modes in equation 1, the central wavelengths of the LPG attenuation bands could be determined as a function of overlay thickness and refractive index, and of the surrounding refractive index.

Figure 3 illustrates the predicted response of the LPG to surrounding refractive index for different overlay thicknesses. The predicted external index response of an LPG with no overlay matches closely the form of the experimentally measured response, shown in figure 2. The graph shows that the presence of the film modifies the range of refractive indices to which the LPG is sensitive, while the sensitivity to lower refractive indices increases with increasing film thickness. It is also shown that, in the presence of the overlay, the LPG becomes sensitive to refractive indices higher than that of the cladding.

The Langmuir Blodgett technique allows the manipulation of materials at the molecular level. A monolayer of the organic material is spread on the subphase of pure water. The molecules are engineered such that one end of the chain is hydrophilic, while the other is hydrophobic. The monolayer is compressed to form an ordered film on the water subphase. A 
substrate passed through the air/water interface picks up a monolayer of thickness approx $3 \mathrm{~nm}$. Repeated passes through the interface allows a film to be built up one molecular layer at a time. High resolution control over the film thickness is achieved, ideal for waveguide applications. The technique may be readily adapted to facilitate uniform deposition of thin films onto a cylindrical structure such as an optical fibre ${ }^{15,16}$.

The material deposited onto the fibres was tricosenoic acid $\left[\mathrm{CH}_{2}=\mathrm{CH}\left(\mathrm{CH}_{2}\right)_{20} \mathrm{CO}_{2} \mathrm{H}\right]$. The material has a molecular length of $2.8 \mathrm{~nm}$, and refractive index 1.57 at $633 \mathrm{~nm}^{17}$. The material was spread from dilute chloroform solutions $(0.1 \mathrm{mg} \mathrm{mL}-1)$ onto the pure water subphase (conductivity $18 \mathrm{M} \Omega . \mathrm{cm}$ ) of one compartment of a Nima Technology Model $2410 \mathrm{~A}$ LB trough, left for 20 minutes at $\sim 20^{\circ} \mathrm{C}$, and compressed at $0.5 \mathrm{~cm}^{2} \mathrm{~s}^{-1}\left(\sim 0.1 \% \mathrm{~s}^{-1}\right.$ of total surface area). Deposition was achieved at a surface pressure of $30 \mathrm{mN} \mathrm{m}^{-1}$ and a transfer rate of $8 \mathrm{~mm} \mathrm{~min}^{-1}$. The fibre containing the LPG was positioned vertically so its long axis was aligned with the dipping direction and was alternately raised and lowered through the floating monolayer at the air-water interface. This procedure gave a Y-type structure in which the amphiphilic molecules were packed head to head and tail to tail. Multiple passes through the film of molecular layer thickness $2.6 \mathrm{~nm}$ were carried out to prepare LPGs with differing film thicknesses, and their response to immersion in Cargille oils of differing refractive indices monitored. The output from a tungsten halogen light bulb was coupled into the optical fiber containing the LPG. The output from the distal end of the fiber was incident on a PC interfaced CCD spectrometer (Ocean Optics S2000). The spectrometer offers a measurement range of $500 \mathrm{~nm}$ to $1100 \mathrm{~nm}$, a resolution of $0.05 \mathrm{~nm}$. The oil was maintained at a constant temperature of $25^{\circ} \mathrm{C}$ using a thermoelectric cooler. 
The measured responses are shown in figure 4 . The form of the results matches the theoretical prediction shown in figure 3, showing the enhancement of the sensitivity to low refractive indices lower than that of silica, and the development of sensitivity to refractive indices higher than that of the silica cladding.

To illustrate the influence of the presence of the thin film upon the performance of the LPG as a chemical concentration sensor, the response of the LPG to immersion in solutions of varying concentration of ethylene glycol was measured before and after the deposition of an overlay of thickness $90 \mathrm{~nm}$. The results are shown in figure 5 . A trend line $\left(4^{\text {th }}\right.$ order polynomial) has been added to each data set as an aid to the eye. The LPG coated with a film of thickness $90 \mathrm{~nm}$ exhibits a larger wavelength shift with increasing concentration of ethylene glycol and concomitant increase in the surrounding refractive index ${ }^{3,18}$. The enhancement in sensitivity increases at higher concentrations, and is approximately double at $100 \%$ ethylene glycol concentration.

In summary, it has been shown, experimentally and using a simple theoretical model, that the deposition of a thin film of material with refractive index higher that that of the cladding onto an LPG can modify the LPG's sensitivity to the external refractive index. Enhanced sensitivity to refractive indices lower than that of the fibre's silica cladding was observed, and the presence of the film resulted in the LPG exhibiting sensitivity to refractive indices higher than that of the cladding.

In the experiments discussed here, the optical properties of the material used to form the overlay were insensitive to the oil. However, it is possible to engineer LB films that 
change their refractive index in response to, for example, exposure to a particular chemical species $^{16}$, offering the prospect for developing a new chemical sensing capability.

The authors would like to acknowledge the support of the Royal Society, UK, the Engineering and Physical Sciences Research Council, UK, and the Spanish Government for supporting Antonio Quintela during his time at Cranfield University. 


\section{References}

1 S.W. James and R.P. Tatam, Optical fibre long-period grating sensors: characteristics and application, Meas.Sci.Technol. 14, R49-R61 (2003)

2 V. Bhatia and A.M. Vengsarakar, Optical fibre long-period grating sensors, Opt.Lett. 21, pp 692-694 (1996).

3 H. J. Patrick, A. D. Kersey and F Bucholtz, Analysis of the Response of Long Period Fibre Gratings to External Index of Refraction, J. Lightwave Technol., 16 pp 1606-42 (1998)

$4 \mathrm{X}$ Shu, and D. Huang, Highly sensitive chemical sensor based on the measurement of the separation of dual resonant peaks in a $100 \mu$ m period fibre grating, Opt.Comm. 171, pp 6569 (1999).

5 T. Allsop, L. Zhang and I. Bennion, Detection of organic aromatic compounds by a long period fibre grating optical sensor with optimised sensitivity, Opt.Comm. 191 pp 181-190 (2001).

6 R. Falciai, A.G. Mignani and A. Vannini, Long period gratings as solution concentration sensors, Sensors and Actuators B 74 pp 74-77 (2001).

7 S. Khaliq, S.W. James and R.P. Tatam, Fibre-optic liquid level sensor using a long period grating, Opt.Lett. 26, pp 1224-1226 (2001).

8 N.J Jang, S.Y. Kim, S.W. Kim and M.S. Kim, Temperature insensitive long-period fibre gratings, Electron. Lett. 35 pp 2134 (1999)

9 S. Yin, K-X Chung and X. Zhu, A novel all-optic tunable long-period grating using a unique double-cladding layer, Opt. Commun. 196 pp181-6 (2001)

10 T. Erdogan, Cladding mode resonances in short and long period fibre grating filters, J.Opt.Soc.Am. 14, pp 1760-1773 (1997), T. Erdogan, Cladding mode resonances in short and long period fibre grating filters, errata J.Opt.Soc.Am. 17, U3-U3 (2000). 
11 V. Bhatia, D.K. Campbell, D. Sherr, T.G. D'Alberto, N.A. Zabaronick, G.A. Ten Eyck, K.A. Murphy and R.O. Claus, Temperature-insensitive and strain insensitive long-period grating sensors for smart structutres, Opt.Eng. 36 pp 1872-1876 (1997).

12 N.D. Rees, S.W. James G.J. Ashwell and R.P. Tatam, Optical fibre long-period gratings with Langmuir-Blodgett thin-film overlays, Opt.Lett. 9, pp 686-688 (2002).

13 A.K. Ghatak, K, Thyagarajan and M.R. Shenoy, Numerical Analysis of Planer Optical Waveguides using Matrix Approach, IEEE J.Lightwave Technol. LT-5, pp660-667 (1987).

14 D. Gloge, Weakly guided fibres, Appl.Opt. 10, pp2252-2258 (1971)

15 R. B. Charters, S. E. Staines and R. P. Tatam, In-Line fibre-optic components using Langmuir-Blodgett Optics Letters, 19 , pp 2036-2038, (1994).

16 D. Flannery, S.W. James, R.P. Tatam and G. Ashwell, Fibre optic chemical sensing using Langmuir-Blodgett overlay waveguides, Appl. Opt. 38, pp 7370-4 ( 1999)

17 K. Skjonnemand, Optical and structural characterisation of ultra thin films, Ph.D. dissertation ( Cranfield University, 2000)

18 D. R. Lide, CRC Handbook of Chemistry and Physics (2004) 


\section{Figure Captions}

Figure 1. Transmission spectrum of an LPG of period $400 \mu \mathrm{m}$, length $40 \mathrm{~mm}$, fabricated in B/Ge co-doped photosensitive optical fibre with cut-off wavelength $650 \mathrm{~nm}$.

Figure 2: Refractive index dependence of the central wavelength of the $5^{\text {th }}$ attenuation band in the spectrum of the LPG detailed in figure 1.

Figure 3. Theoretical prediction of the response of an LPG to the external refractive index. with no overlay, + overlay thickness $75 \mathrm{~nm}$ and $\boldsymbol{\Delta}$ overlay thickness $125 \mathrm{~nm}$. The overlay refractive index is assumed to be 1.57 in the calculations.

Figure 4. Experimentally determined surrounding refractive index response of the $5^{\text {th }}$ attenuation band in the spectrum of the LPG to the external refractive index. a with no overlay; with $90 \mathrm{~nm}$ overlay; $\boldsymbol{\Delta}$ with $120 \mathrm{~nm}$ overlay and - with overlay thickness of $150 \mathrm{~nm}$

Figure 5. Experimentally determined response of the $5^{\text {th }}$ attenuation band in the spectrum of the LPG to the change in concentration of ethylene glycol and refractive index with a no overlay; with overlay thickness $90 \mathrm{~nm}$ 


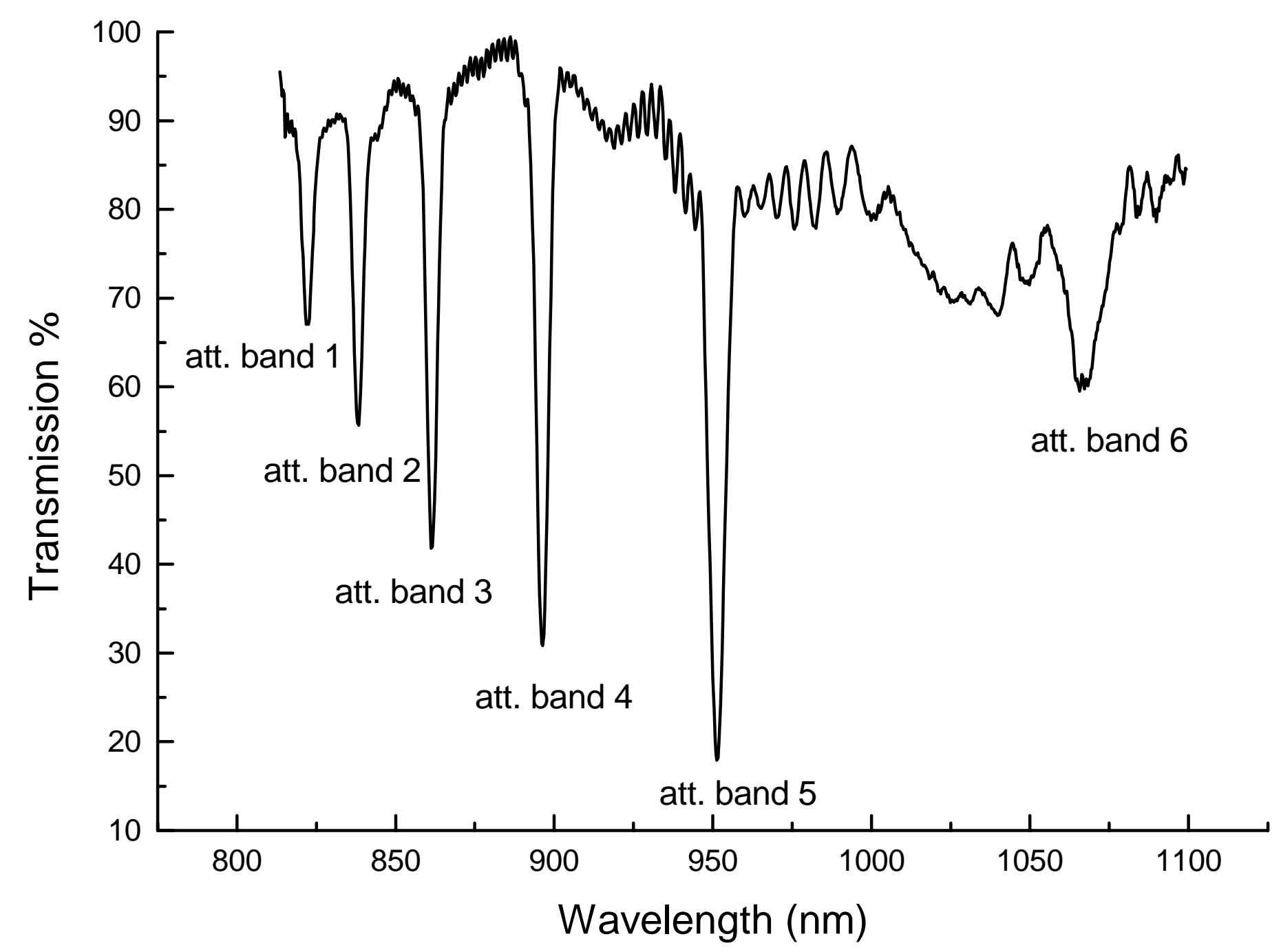

Figure 1 


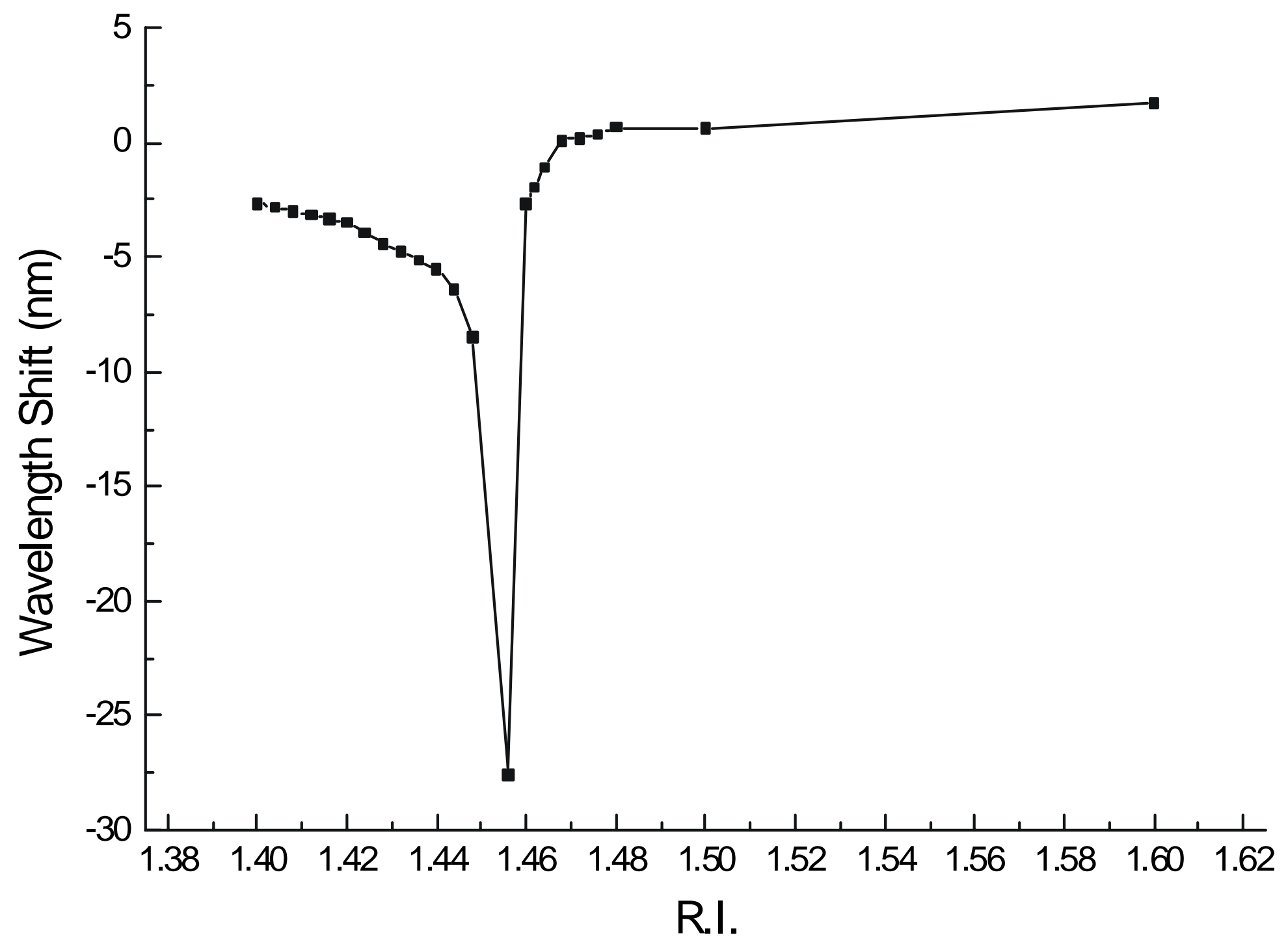

Figure 2 


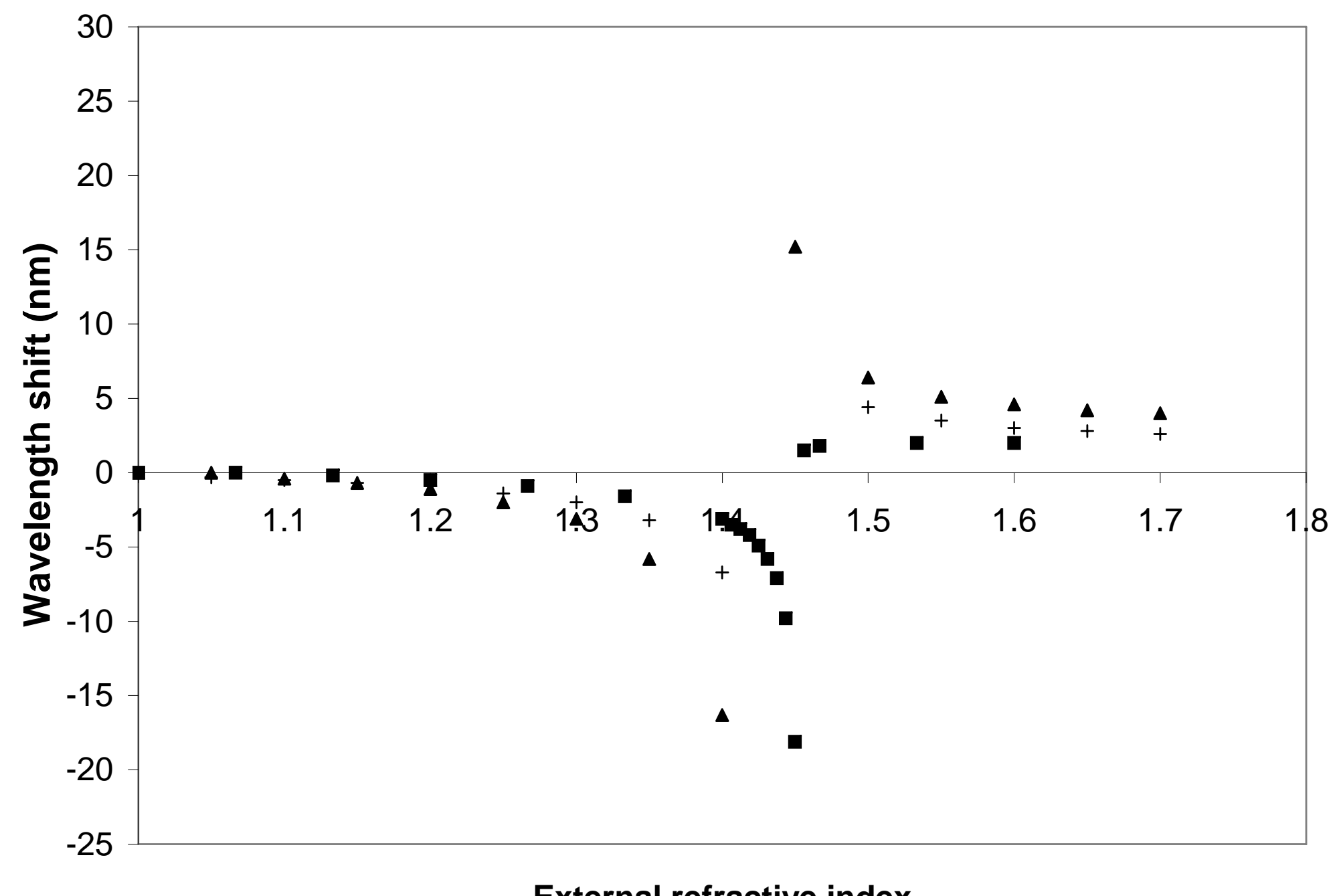

Figure 3 


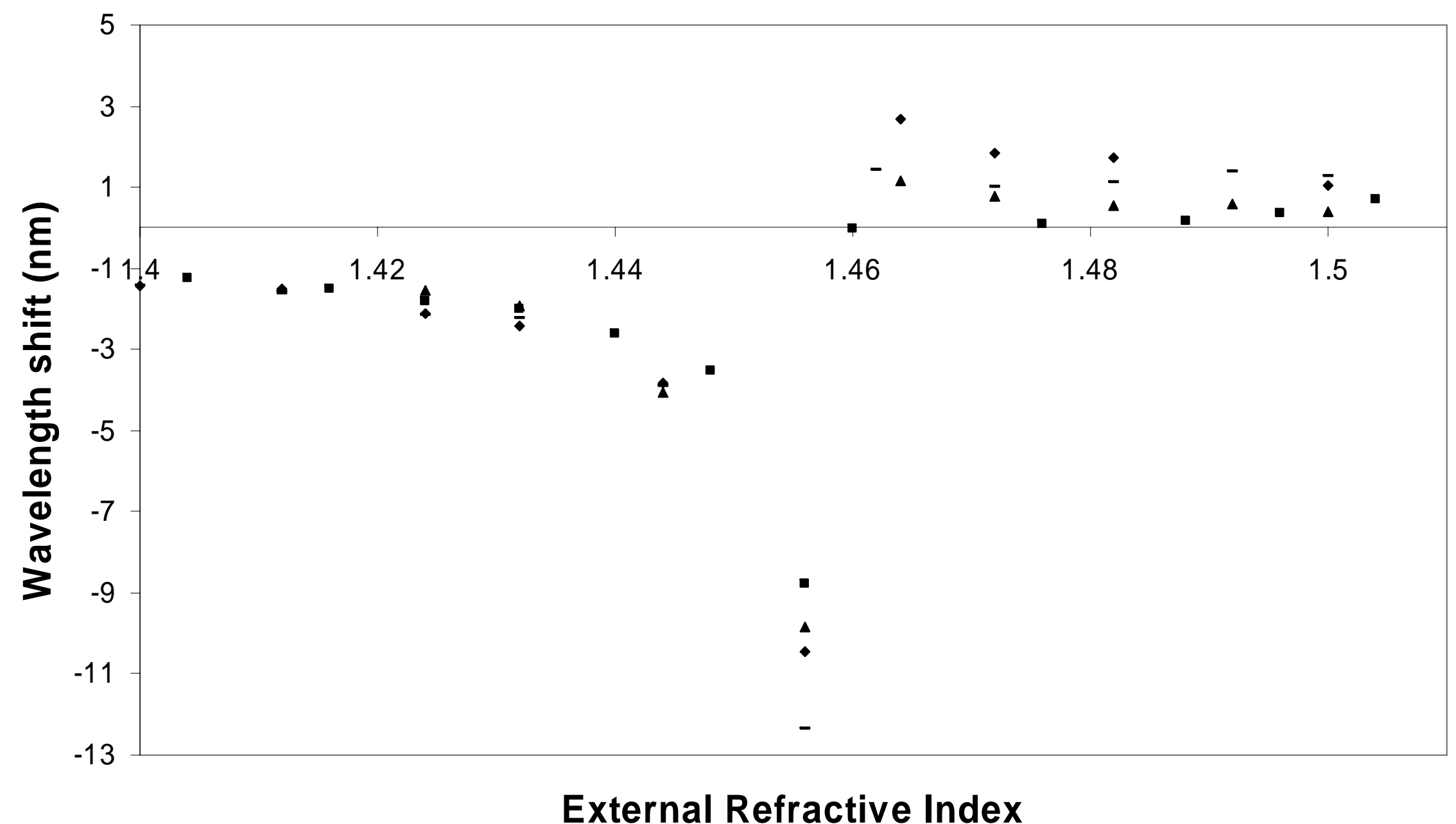

Figure 4 


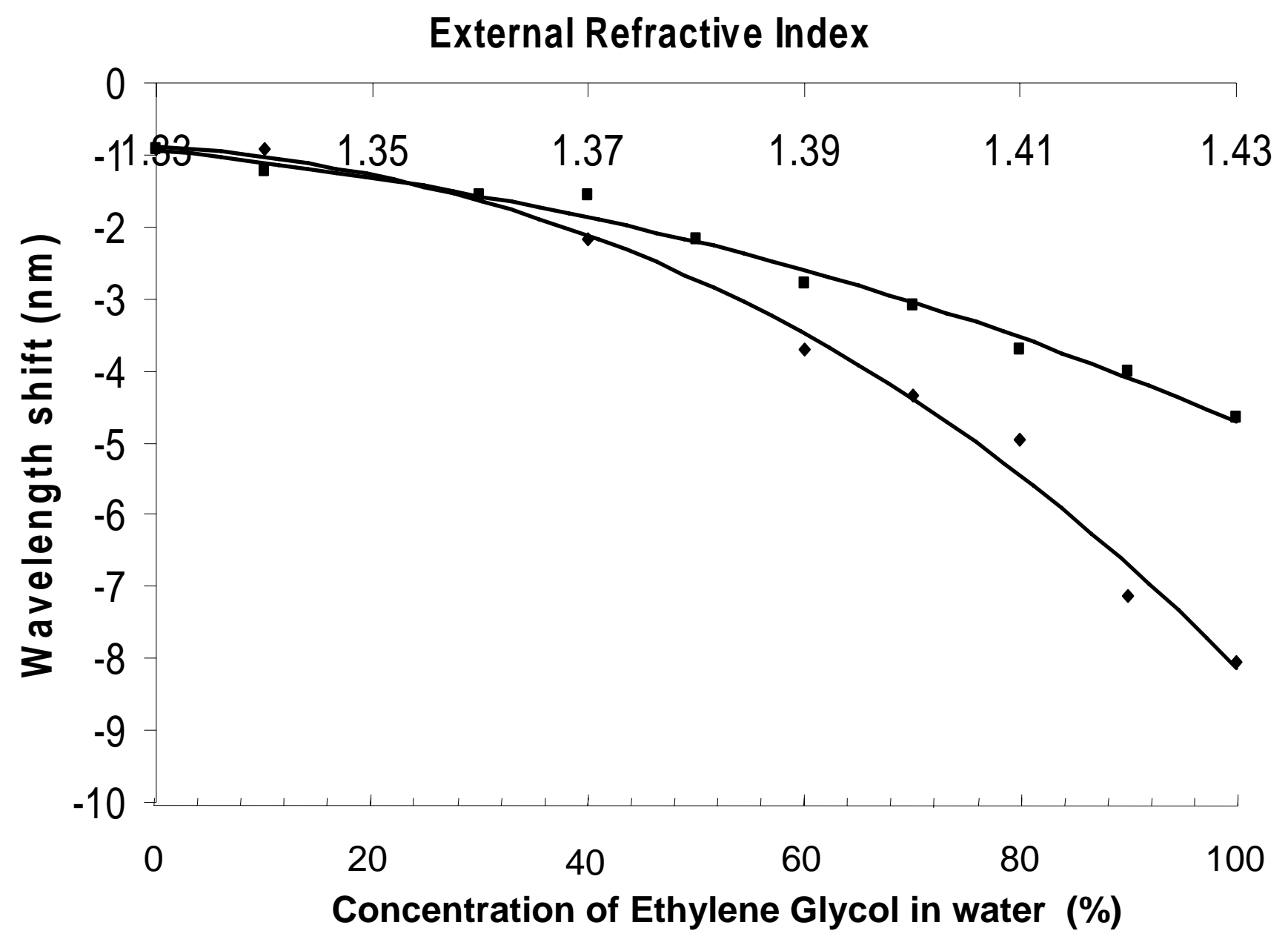

Figure 5 
